\title{
Tratamento endovascular da síndrome da aorta média causada por arterite de Takayasu: relato de caso
}

\author{
Endovascular treatment of the middle aortic syndrome due to \\ Takayasu's arteritis: case report
}

Felipe Nasser ${ }^{1}$, Joaquim Maurício da Motta-Leal-Filho ${ }^{1}$, Francisco Cesar Carnevale ${ }^{1}$, Caio Cesar
Martins Focassio $^{1}$, Dênis Szejnfeld ${ }^{1}$, Rodrigo Oliveira ${ }^{2}$, José Nery Praxedes ${ }^{2}$, Giovanni Guido Cerri ${ }^{1}$

\section{Resumo}

Síndrome da aorta média (SAM) é uma condição clínica caracterizada por estenose segmentar ou difusa da aorta, hipertensão arterial e claudicação de membros inferiores. A principal causa da SAM é a arterite de Takayasu. A estenose segmentar pode estar localizada na aorta suprarrenal, renal ou infrarrenal e com alta propensão de lesões estenóticas concomitantes das artérias renais e viscerais. A gravidade da hipertensão arterial é a principal indicação para o tratmento. A técnica endovascular para o tratamento da síndrome da aorta média tem bons resultados e é a menos invasiva. No presente artigo, é descrito o tratamento com sucesso de uma estenose da aorta toracoabdominal com recanalização da artéria mesentérica superior através de angioplastia em uma mulher de 34 anos portadora de arterite de Takayasu e hipertensão arterial grave.

Palavras-chave: Arterite de Takayasu, angioplastia com balão, stents, aorta abdominal, obstrução da artéria renal, artérias viscerais.

\section{Introdução}

A arterite de Takayasu (AT) é uma doença vascular inflamatória crônica de etiologia desconhecida que se caracteriza por provocar lesões definitivas nos grandes vasos, ramos diretos da aorta, principalmente os supra-aórticos, podendo acometer inclusive a artéria pulmonar. Predomina em mulheres (9:1) jovens (15-25 anos) de descendência asiática. As lesões da aorta e seus ramos podem ser aneurismáticas ou estenótico-oclusivas ${ }^{1-3}$. Das artérias parietais, a mais acometida é a artéria subclávia esquerda e, das viscerais, as artérias renais. Daí a associação da AT com a hipertensão renovascular. O tratamento está baseado:

1) no controle da atividade da doença através dos imunossupressores;

2) na correção cirúrgica nas lesões estenótico-oclusivas longas ou quando há o acometimento de vários ramos viscerais;

\begin{abstract}
Middle aortic syndrome (MAS) is a clinical condition characterized by segmental or diffuse narrowing of the aorta, hypertension, and lower limb claudication. The main cause of MAS is Takayasu's arteritis. Segmental aortic stenosis may be located at the suprarenal, renal or infrarenal aorta with high tendency to concomitant stenosis in both the renal and visceral arteries. Severity of hypertension is the primary indication for intervention. Endovascular therapy is a minimally invasive treatment for MAS and may provide good results. In the present report, we describe a successful endovascular treatment of stenosis of the thoracoabdominal aorta with recanalization of the superior mesenteric artery using angioplasty in a 34-year-old woman with Takaysu's arteritis and severe hypertension.
\end{abstract}

Keywords: Takayasu's arteritis, balloon angioplasty, stents; abdominal aorta, renal artery obstruction, celiac artery.

3) ou no tratamento endovascular através de angioplastia e/ou implante de stent ${ }^{1,3-5}$.

A terapêutica endovascular é efetiva no tratamento da estenose da aorta e seus ramos parietais e viscerais, principalmente nas lesões ostiais ${ }^{3}$. É sabido também que a progressão da doença é responsável pelas altas taxas de recidiva precoce, principalmente em se tratando das artérias renais $^{1,3,6}$.

O portador de AT pode cursar com manifestações clínicas diversas a depender da localização do comprometimento arterial. As lesões podem ser classificadas em quatro tipos: tipo I (Shimizu-Sano), envolvimento do arco aórtico e seus ramos; tipo II (Kimoto), envolvimento da aorta torácica descendente e da aorta abdominal sem comprometimento do arco aórtico; tipo III, comprometimento arterial dos tipos I e II; tipo IV, comprometimento arterial

1. Serviço de Radiologia Intervencionista, Hospital das Clínicas, Faculdade de Medicina da Universidade de São Paulo (FMUSP), São Paulo, SP.

2. Serviço de Nefrologia, Hospital das Clínicas, FMUSP, São Paulo, SP.

Não foram declarados conflitos de interesse associados à publicação deste artigo.

Artigo submetido em 13.07.08, aceito em 25.09.09.

J Vasc Bras. 2009;8(4):359-363.

Copyright $@ 2009$ by Sociedade Brasileira de Angiologia e de Cirurgia Vascular 
dos tipos I, II ou III, adicionando-se o acometimento da artéria pulmonar ${ }^{7}$. Quando o comprometimento da aorta toracoabdominal e/ou da aorta abdominal leva a um quadro de hipertensão arterial sistêmica associado à diminuição dos pulsos femorais e claudicação dos membros inferiores denomina-se síndrome da aorta média $(\mathrm{SAM})^{4,5,8-12}$. A obstrução mecânica da aorta parece ser a causa da hipertensão arterial.

O presente artigo tem como objetivo relatar um caso de tratamento endovascular de estenose da aorta toracoabdominal e recanalização da artéria mesentérica superior através de angioplastia com balão em paciente portadora de AT tipo III ${ }^{1,7}$.

\section{Descrição do caso}

Paciente do sexo feminino, 34 anos, assintomática, com diagnóstico de AT controlada através do uso contínuo de imunossupressores (prednisona $5 \mathrm{mg}, 2 / 2$ dias e metotrexate $25 \mathrm{mg} / \mathrm{semana}$ ). Negava sintomas compatíveis com claudicação de membros superiores, membros inferiores e gastrointestinais. Estava em tratamento de hipertensão renovascular usando três drogas (atenolol 100 mg/dia, amlodipina $10 \mathrm{mg} /$ dia e clortalidona $25 \mathrm{mg} / \mathrm{dia}$ ). Exame físico: sopro na artéria carótida esquerda, em ambas as artérias subclávias e no flanco esquerdo. Pulsos femorais diminuídos $(+2 /+4)$ e simétricos. Pressão arterial (PA): $170 \mathrm{x}$ $80 \mathrm{mmHg}$ no membro superior direito e 160 x $80 \mathrm{mmHg}$ no membro superior esquerdo. Angiotomografia realizada dois anos antes evidenciava lesão estenótica na artéria subclávia esquerda (ASE), tronco braquiocefálico (TBC), artéria carótida comum esquerda (ACCE), aorta toracoabdominal (ATA), artéria mesentérica superior (AMS) e artérias renais (AR). A angiotomografia atual demonstrava piora das lesões nas artérias AMS, artéria renal esquerda (ARE) e ATA (Figuras 1 e 2). As provas de atividade inflamatória - proteína $\mathrm{C}$ reativa $=1,64 \mathrm{mg} / \mathrm{L}($ normal $<3,0)$ e velocidade de hemossedimentação $=14 \mathrm{~mm} / \mathrm{h}$ (normal $<12$ em mulher) -, sem alterações significativas, demonstravam controle da doença; e a função renal era preservada (creatinina $0,6 \mathrm{mg} / \mathrm{dL}$ e clearance de creatinina de $102 \mathrm{~mL} / \mathrm{min}$ ). Diante das importantes lesões estenóticas e do quadro hipertensivo da paciente, optou-se por realizar a terapêutica endovascular.

Técnica: obteve-se o acesso arterial femoral direito, sob anestesia local, pela técnica de Seldinger. Adminis- trou-se leve sedação pela equipe de anestesia, não havendo necessidade de anestesia geral. Realizou-se aortografia com cateter pigtail centimetrado posicionada na aorta torácica distal, confirmando-se a lesão estenótica na ATA, a não opacificação da AMS, estenose moderada das AR e estenose moderada da aorta abdominal infrarrenal (AAIR)

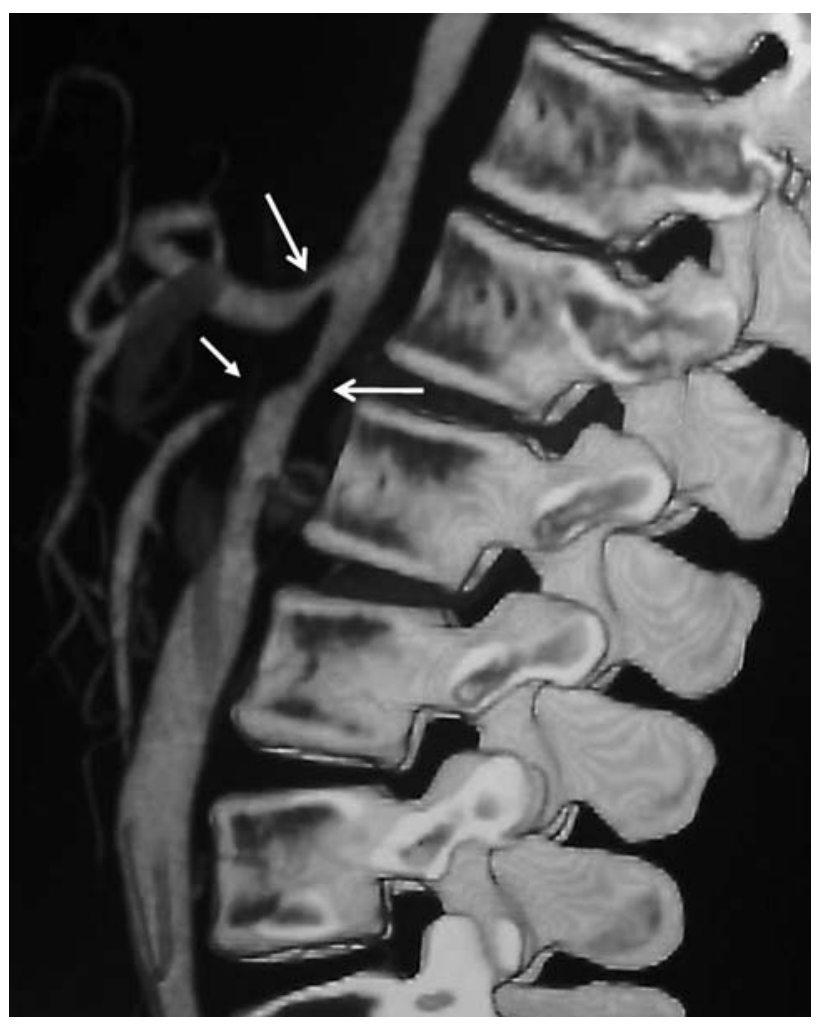

Figura 1 - Reconstrução angiotomográfica da aorta toracoabdominal mostrando estenose grave da aorta e da artéria mesentérica superior e estenose moderada do tronco celíaco.

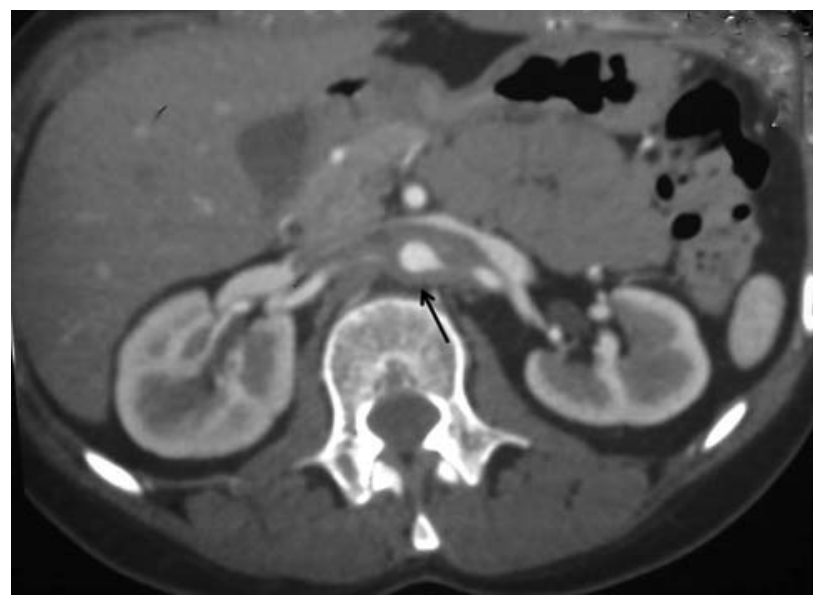

Figura 2 - Corte axial tomográfico da aorta abdominal ao nível das artérias renais mostrando a espessura da parede arterial 
(Figura 3). O gradiente pressórico sistólico translesional aórtico (proximal ao nível do diafragma e distal ao nível da bifurcação das artérias ilíacas) foi de $50 \mathrm{mmHg}$. Realizou-se a heparinização sistêmica na dose de $100 \mathrm{UI} / \mathrm{kg}$ seguida da angioplastia da ATA com cateter-balão $10 \mathrm{x}$ $40 \mathrm{~mm}$ (Ultrathin Diamond, Boston Scientific, Natick, EUA) (Figura 4). O tempo de insuflação do balão foi de 3 minutos com pressão máxima de $15 \mathrm{~atm}$. A angiografia pós-tratamento evidenciou melhora da lesão aórtica e opa-

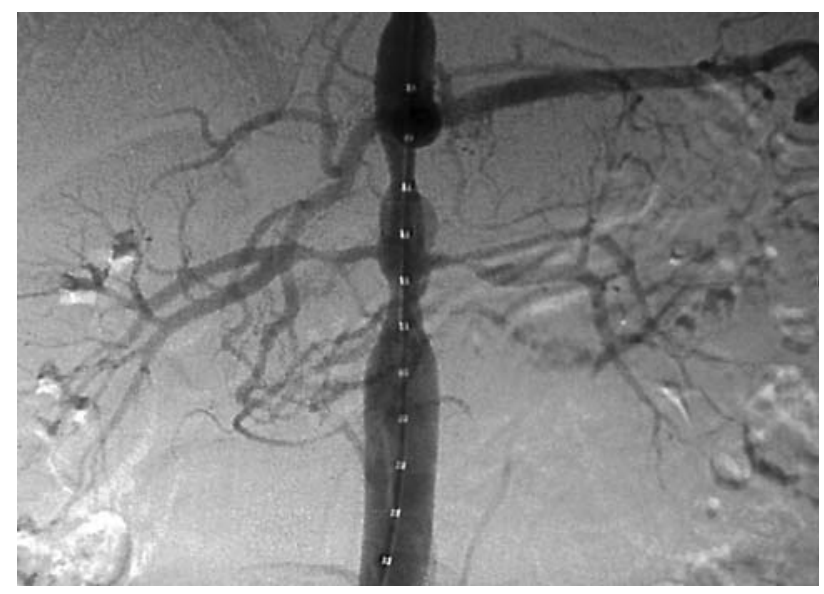

Figura 3 - Arteriografia com subtração digital mostrando a estenose segmentar na aorta toracoabdominal

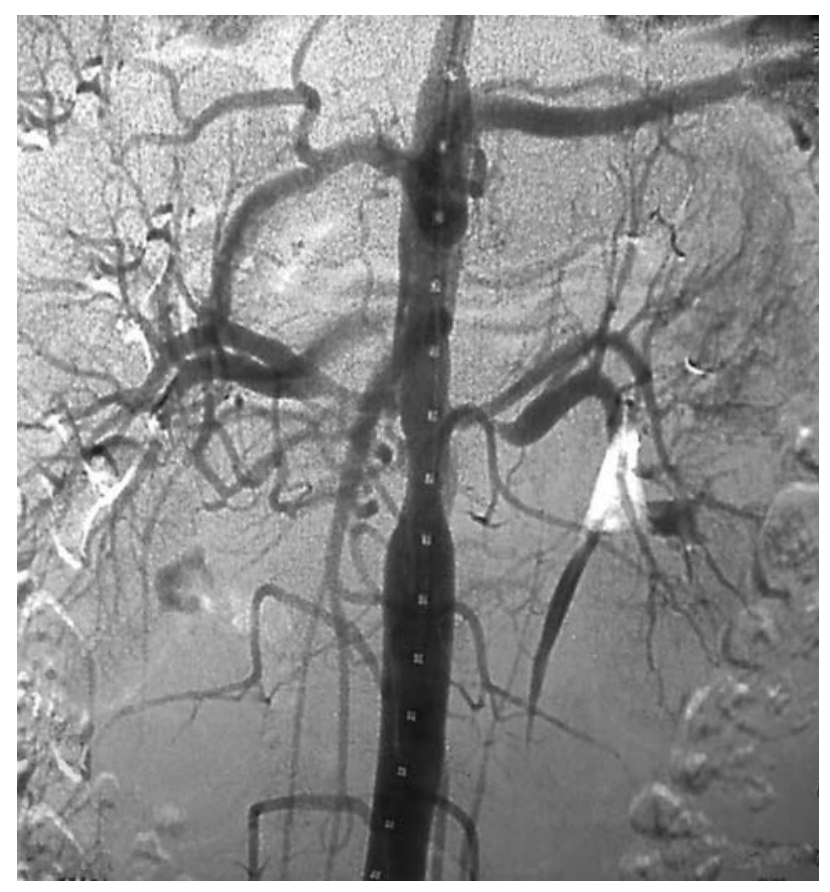

Figura 4 - Arteriografia com subtração digital pós-angioplastia com balão mostrando o tratamento da estenose da aorta toracoabdominal com opacificação da artéria mesentérica superior cificação da AMS. Observou-se queda do gradiente pressórico translesional de 50 para $10 \mathrm{mmHg}$, demonstrando o tratamento efetivo da lesão aórtica. As AR não foram tratadas. Na evolução pós-operatória imediata obteve-se queda dos níveis pressóricos nos membros superiores (PA $130 \mathrm{x}$ $80 \mathrm{mmHg}$ ) e diminuição da medicação anti-hipertensiva (atenolol $50 \mathrm{mg} /$ dia e amlodipina $5 \mathrm{mg} / \mathrm{dia}$ ). A paciente manteve-se assintomática, obtendo alta hospitalar no terceiro dia em uso de ácido acetilsalicílico (AAS) $200 \mathrm{mg} /$ dia e de clopidogrel $75 \mathrm{mg} /$ dia, este último mantido por seis meses, além do uso das medicações habituais anti-hipertensivas e imunossupressoras. Atualmente encontra-se no $10^{\circ}$ mês pós-operatório, com os seus níveis pressóricos controlados somente com atenolol $25 \mathrm{mg} /$ dia e em uso de AAS $200 \mathrm{mg} /$ dia, prednisona $5 \mathrm{mg}-2 / 2$ dias e metotrexate $25 \mathrm{mg} / \mathrm{semana}$.

\section{Discussão}

A SAM, acometimento localizado ou difuso da aorta toracoabdominal, é uma causa incomum de hipertensão arterial e claudicação dos membros inferiores ${ }^{4,5,8,9}$. Sua etiopatogenia é desconhecida e controversa. Alguns autores advogam que sua origem é congênita, devendo-se à falha de fusão do par aórtico dorsal na quarta semana de gestação ${ }^{4,8-12}$. Outros defendem que é um quadro associado à aortite. De fato, a síndrome está associada a certas doenças, como arterite de Takayasu, neurofibromatose, fibrose retroperitoneal e mucopolissarcoidose $e^{4,8-12}$.

No presente caso, trata-se de uma paciente portadora de AT há 10 anos (preenche quatro critérios diagnósticos pela classificação do Colégio Americano de Reumatologia e preenche critérios diagnósticos de Ishikawa) ${ }^{2,13}$ e com hipertensão arterial há 3 anos. Não apresentava claudicação nos membros inferiores, mas não realizava atividade física regular e tinha pulsos femorais diminuídos, porém simétricos. Estava em tratamento clínico para AT. Este tratamento já está bem estabelecido na literatura, utilizando-se imunossupressores como a prednisona e/ou metotrexate no intuito de diminuir ou eliminar a atividade inflamatória ${ }^{1-3}$. Os imunossupressores devem ser mantidos mesmo quando os marcadores desta atividade estiverem normais. As abordagens cirúrgica e endovascular só devem ser realizadas quando não houver atividade inflamatória, algo conseguido através do uso contínuo das drogas imunossupressoras. Caso contrário, estes tratamentos se tornam arriscados e, 
se realizados na vigência de atividade inflamatória, têm piores resultados ${ }^{3,14}$.

O tratamento endovascular parece controverso na literatura. Muitos autores utilizam a angioplastia com implante de stent, pois acreditam que, dessa forma, diminuem as taxas de complicações (dissecção e reestenose) e aumentam o tempo de permeabilidade do vaso ${ }^{1,3,5}$. Outros autores utilizam o cutting balloon por acreditarem se tratar de lesões de natureza endurecida e resistente ${ }^{3}$. No entanto, deve-se ressaltar que o diâmetro máximo do cutting balloon é de $8 \mathrm{~mm}$ e nem todo paciente possui uma lesão aórtica compatível com seu uso. Optou-se por angioplastia com cateter-balão sem implante de stent por se tratar de uma aorta de $10 \mathrm{~mm}$ de diâmetro e com lesão estenótica localizada entre os ramos viscerais da ATA (tronco celíaco, AMS e AR). O implante do stent nesta topografia resultaria na dificuldade técnica de nova abordagem destes ramos viscerais ou mesmo poderia resultar na oclusão destes ramos. Vale ressaltar que se trata de uma doença inflamatória progressiva que comumente requer outros procedimentos endovasculares para manutenção da permeabilidade dos vasos tratados ${ }^{1,3,6}$. Na arteriografia de controle pósangioplastia da ATA observou-se a opacificação da AMS. Concluiu-se que havia uma sobreposição de estenoses (estenose grave na ATA e outra ostial leve na AMS) e que o tratamento dado à ATA tinha proporcionado um aumento do fluxo na AMS, restaurando o fluxo arterial normal, deixando de ser significativa e não havendo necessidade de angioplastia desta última.

Apesar de a paciente possuir lesões estenóticas nas $\mathrm{AR}$, principalmente à esquerda, optou-se por tratamento somente da aorta por se tratar de uma hipertensão causada pela estenose da ATA (SAM), fato este corroborado pela queda do gradiente pressórico imediatamente após angioplastia transluminal percutânea (de $50 \mathrm{mmHg}$ para $10 \mathrm{mmHg}$ ), queda da pressão arterial sistêmica e diminuição da medicação anti-hipertensiva subsequente.

O tratamento cirúrgico convencional é factível e é indicado principalmente quando as lesões aórticas são extensas, mas possui maior morbimortalidade $\mathrm{e}^{1,3,8,9}$.

O acompanhamento pós-operatório desses pacientes deve ser com consultas de rotina, monitoramento da pressão arterial, provas de atividade inflamatória e exames de imagens da aorta e seus ramos para diagnosticar novas lesões estenóticas passíveis de tratamento, já que mesmo com a doença controlada clinicamente as lesões arteriais podem continuar progredindo e sendo necessário outras intervenções ${ }^{1,3,5,6,14}$.

A SAM causada por arterite de Takayasu é uma doença extremamente grave e progressiva. $\mathrm{O}$ tratamento endovascular é uma boa opção de tratamento, pois reúne a vantagem de ser uma técnica minimamente invasiva e a possibilidade de novas reintervenções e com bons resultados imediatos, além de permitir ao paciente o retorno precoce a suas atividades. Devido ao seu caráter progressivo, os pacientes que sofrem desse mal devem ter a doença controlada através de medicações anti-inflamatórias e/ou imunossupressoras, ser monitorados com angiotomografias periódicas e reabordados quando necessário.

\section{Referências}

1. Kauffman P, Sitrângulo CJ. Arterites de células gigantes. In: Maffei FH, editor. Doenças vasculares periféricas. $3^{\mathrm{a}}$ ed. Rio de Janeiro: MEDSI; 2002. p. 1281-9.

2. Arend WP, Michel BA, Bloch DA, et al. The American College of Rheumatology 1990 criteria for the classification of Takayasu arteritis. Arthritis Rheum. 1990;33:1129-34.

3. Joseph G, George PV, Pati PK, Chandy ST. Feasibility of angioplasty and stenting for abdominal aortic lesions adjacent to previously stented visceral artery lesions in patients with Takayasu arteritis. Cardiovasc Intervent Radiol. 2007;30:293-6.

4. Perrone-Filardi P, Costanzo P, Cesarano P, et al. Long abdominal aortic stenosis: a rare presentation of Takayasu arteritis treated with percutaneous stent implantation. J Thorac Cardiovasc Surg. 2007;133:1647-8.

5. Bialkowski J, Szkutnik M, Bermúdez-Cañete R, Kusa J, Regiec S, Mullins CE. Middle aortic syndrome caused by Takayasu's disease and treated by stent implantation: a report of medium-term follow-up. Rev Esp Cardiol. 2002;55:682-5.

6. Giordano JM, Hoffman GS. Takayasu's disease: nonspecific aortoarteritis. In: Rutherford RB, editor. Vascular surgery. $5^{\text {a }}$ ed. Philadelphia: WB Saunders; 2000. p. 364-73.

7. Lupi-Herrera E, Sánchez-Torres G, Marcushamer J, Mispireta J, Horwitz S, Vela JE. Takayasu's arteritis. Clinical study of 107 cases. Am Heart J. 1977;93:94-103.

8. Adovasio R, Canci U. Middle aortic syndrome: an atypical case - a case report. Angiology. 2000;51:525-8.

9. Seki A, Ogawa H, Fujiu K, Kawagoe Y, Kasanuki H. Middle aortic syndrome diagnosed at 54 years of age - a case report. Angiology. 2002;53:605-8.

10. Sen PK, Kinare SG, Engineer SD, Parulkar GB. The middle aortic syndrome. Br Heart J. 1963;25:610-8.

11. Daimon S, Kitamura K. Coarctation of the abdominal aorta. Jpn Heart J. 1964;5:562-73.

12. Lande A. Takayasu's arteritis and congenital coarctation of the descending thoracic and abdominal aorta: a critical review. AJR Am J Roentgenol. 1976;127:227-33. 
13. Ishikawa K. Diagnostic approach and proposed criteria for the clinical diagnosis of Takayasu's arteriopathy. J Am Coll Cardiol. 1988;12:964-72.

14. Park MC, Lee SW, Park YB, Lee SK, Choi D, Shim WH. Post-interventional immunosuppressive treatment and vascular restenosis in Takayasu's arteritis. Rheumatology (Oxford). 2006;45:600-5.

Correspondência:

Joaquim Maurício da Motta Leal Filho

Rua Diogo Jacome, 1030/121, Vila Nova Conceição CEP 04512-001 - São Paulo, SP

Tel.: (11) 5052.9028, (11) 9974.7794

E-mail: jotamauf@yahoo.com.br,jmaufi@gmail.com

\section{Contribuições dos autores:}

Concepção e desenho do estudo: FN, JMMLF, FCC

Análise e interpretação dos dados: FN, JMMLF

Coleta de dados: JMMLF, CCMF, DS, RO, JNP

Redação do artigo: JMMLF

Revisão crítica do texto: FN, JMMLF, FCC,RO, JNP, GGG

Aprovação final do artigo*: FN, JMMLF, FCC, CCMF, DS, RO, JNP, GGG

Análise estatística: JMMLF

Responsabilidade geral pelo estudo: FN, JMMLF, FCC, JNP, GGG

Informações sobre financiamento: N/A

*Todos os autores leram e aprovaram a versão final submetida ao J Vasc Bras. 